complete or timely way using other methods. Equally, the conditions to be studied must have clear importance to the ophthalmological community for at least one of the three following reasons: firstly, they are significant for public health or health policy; secondly, their study has important direct implications for clinical practice; and, thirdly, they offer the opportunity to study fundamental disease mechanisms. These requirements are illustrated by the range of studies currently being facilitated by BOSU. A study of Acanthamoeba keratitis and its relation to soft contact lens wear is a timely examination that has direct relevance to public health and clinical practice. Similarly, a study of the incidence and causes of loss of vision in the non-amblyopic eye amongst individuals with unilateral amblyopia addresses topical, unanswered questions important to the debate about childhood vision screening. By contrast, a study of sympathetic ophthalmia provides a new opportunity to characterise and clarify pathogenesis in a representative range of cases. Finally, a study of retinopathy of prematurity, to determine incidence and outcomes in a national cohort of affected children, will also gather information of importance to future provision of screening services for this disorder.

BOSU offers the ophthalmological community a new and effective medium for improving the study, thereby the understanding, and ultimately the management, of a variety of important but rare ophthalmic disorders. Everyone involved in clinical, basic scientific, or epidemiological research on such disorders is encouraged to consider this new service. The success of BOSU, and the studies it supports, depends on the continued interest and involvement of the reporting clinicians who, together with their patients, it ultimately serves. It is hoped that the ophthalmological community will continue to support this initiative to enhance the research base of ophthalmology.

J. R. is supported by the Medical Research Council.

Department of Epidemiology J. S. RAHI*

Institute of Child Health

London

Ipswich Hospital NHS Trust

C. EdELSTEN*

Ipswich

Suffolk

*On behalf of the Steering Committee of the British Ophthalmological Surveillance Unit.

\section{References}

1. Stanford M. Br J Ophthalmol 1997;81:932-3.

2. Langmuir AD. The surveillance of communicable diseases of national importance. N Engl J Med 1963; 268:182-91.

3. Farr W. Cholera epidemic, 1848-49. In: Humphreys NA, editor. Vital statistics: a memorial volume of selections from the reports and writings of William Farr. London: E Sanford, 1885:333-4.

4. British Paediatric Surveillance Unit. Lancet 1992;340: 845.

5. Cockerell OC, Gupta S, Catchpole M, Sander JW, Shorvan SD. The British Neurological Surveillance Unit: a nationwide scheme for the ascertainment of rare neurological disorders. Neuroepidemiology 1995;14:182-7.

6. Gilbert RE, Stanford MR, Jackson H, Holliman RE, Sanders MD. The incidence of acute symptomatic Toxoplasma retinochoroiditis in south London according to country of birth. BMJ 1995;310:1037-40.

7. The British Congenital Cataract Study. British Paediatric Surveillance Unit Annual Report 1996. London: Royal College of Paediatrics and Child Health, 1997.

\title{
HOW SHOULD ACTIVE TOXOPLASMIC RETINOCHOROIDITIS BE TREATED?
}

Despite a plethora of publications concerning Toxoplasma and toxoplasmosis,${ }^{1}$ clinical diagnosis of the ocular disease remains subjective, and treatment largely empirical. This is due, at least in part, to a lack of consistent data pertaining to the host-parasite relationship within the human uveo-retinal tract, and particularly whether, in vivo, Toxoplasma is actually responsible for initiation and perpetuation of the ocular disease. Such knowledge is necessary if there is to be a rational approach to the medical therapy of ocular toxoplasmosis. ${ }^{2}$

The Toxoplasma tissue cyst may be the major contributor to the ongoing pathogenetic process. Electron microscopy of animal ocular tissue with optimal fixation has revealed that intact tissue cysts in the retina are invariably located within viable cells in the ganglion cell and/or inner nuclear layers. ${ }^{3}$ Demise of the host cell liberates viable toxoplasms and other Toxoplasma antigens into the surrounding host tissue, to elicit a localised inflammatory event and, perhaps, permitting parasitisation of adjacent cells. Activated macrophages and other immune cells process the Toxoplasma antigen, ${ }^{4}$ thus suppressing the infection. Medical intervention is usually necessary, however, to reduce progression of inflammation, especially if the lesion is considered clinically to be sight-threatening. ${ }^{5}$

Proposed chemotherapeutic strategies for toxoplasmic retinopathy, ${ }^{6}$ including cases associated with pregnancy, neonates and the severely immunocom- 
promised, ${ }^{7}$ should ideally eliminate free forms of Toxoplasma prior to their re-invasion of host cells, as well as parasites enclosed within intact tissue cysts. Moreover, ocular disease should not be exacerbated as a consequence of drug intervention. The mainstay of toxoplasmic retinochoroiditis therapeutics in all categories of patient has been the folate synthesis inhibitors, primarily pyrimethamine and sulphadiazine. These, and the protein biosynthesis inhibitor clindamycin, are effective against the extra-cystic form of Toxoplasma only. ${ }^{8}$ Thus, on a theoretical basis at least, these drugs are effective in vivo only during a relatively short period directly after the onset of a clinically perceived inflammatory episode. Immediate treatment with such agents at the onset of symptoms thus represents a logical approach to chemotherapy. Such intervention could reduce the likelihood of re-invasion by Toxoplasma of other host cells and, in theory at least, both shorten the disease process and reduce the likelihood of subsequent extension and recurrence, by eliminating extracellular parasites before they penetrate adjacent host cells.

It remains to be established whether these drugs remain effective after an inflammatory episode is established. Only one publication provides data on this: a prospective multicentre controlled trial of folate antagonists and clindamycin in the context of treatment of ocular toxoplasmosis. ${ }^{9}$ In this study use of the drugs did not result in benefits to the patient in terms of reducing duration or severity of the perceived inflammatory episode, but pyrimethamine may have reduced the area of focal scarring, an observation which could, however, have been due to chance. Furthermore, the trial was not randomised or masked, and these factors may have influenced the outcome. Rational treatment strategies, for both immunocompetent and immunocompromised individuals, thus remain to be established. A recent longitudinal study ${ }^{10}$ aimed at assessing the features of the eye disease in early congenital toxoplasmosis, suggests that active lesions may become quiescent with pyrimethamine and sulphadiazine therapy.

Ocular toxoplasmosis in individuals infected with human immunodeficiency viruses provides a singular challenge to the ophthalmologist. Pyrimethamine and sulphadiazine (and clindamycin) are again the mainstay of treatment in this high-risk group. ${ }^{11}$ Individual resolutions have been recorded with other drugs such as tetracycline and spiramycin ${ }^{12}$ in patients with AIDS. Clearly, a rational approach to chemotherapy, such as is available for treatment of toxoplasmic encephalitis,$^{13}$ would be beneficial in the ocular setting. Furthermore, the potential role of other anti-Toxoplasma drugs including azithromycin and other newer macrolides, and the hydroxynaphthoquinone, atovaquone, with or without con- current corticosteroid therapy, requires to be evaluated.

Systemic corticosteroids are commonly used as part of the medical treatment of active episodes of toxoplasmic retinochoroiditis. ${ }^{6}$ Steroid therapy was introduced into Toxoplasma therapeutics shortly after the observation that focal inflammation and scarring were associated with the infection. The rationale for steroid use in the setting of active toxoplasmic retinochoroiditis is in prevention or reduction of retinal tissue damage by the so-called innocent bystander mechanism. There is histological evidence to support this effect, but whether steroid use actually does yield any therapeutic benefit to the patient remains controversial, and clearly requires further investigation. Pharmacological immunosuppression with corticosteroids can enhance the progression of a Toxoplasma infection in laboratory animals, with increased proliferation of the protozoa within host tissues.

Of particular relevance to the outcome of ocular toxoplasmosis is that at therapeutic concentrations glucocorticoids, including progesterone, can markedly suppress the antimicrobial activity of macrophages, even though they do not necessarily inhibit macrophage activation by cytokines. For some intracellular microbes this effect can be reduced by interferon-gamma (IFN- $\gamma)$. Although some cytokines (e.g. interleukin-6) may be associated with an increase in the inflammatory response to Toxoplasma ${ }^{14}$ others (e.g. IFN- $\gamma$, tumour necrosis factor-alpha, interleukin-2, interleukin-12) are important in resistance against Toxoplasma. ${ }^{15}$ The last produces this effect by, for example, stimulation of macrophages and/or natural killer cells. Thus there may be a therapeutic role for cytokines in patients with toxoplasmic encephalitis. Utilisation of cytokines in the immunosuppressed, or in immunoprivileged sites such as the eye, however, may prove impracticable, thus precluding their therapeutic use in ophthalmology. Reduction in IFN- $\gamma$ or tumour necrosis factor-alpha in mice with post-natally acquired ocular toxoplasmosis actually increased the number of ocular lesions and the severity of the inflammatory response. ${ }^{16}$

Glucocorticoids can act as regulators of the immune response and can suppress the synthesis of, for example, IFN- $\gamma \cdot{ }^{17}$ Moreover, CMI response components can be suppressed by glucocorticoids through the induction of lymphocytopenia and monocytopenia, and through interference with the early stages of proliferation and differentiation of the immune response. Direct cytotoxicity in a small subset of activated lymphocytes can permit glucocorticoids to impair CMI. These events can increase the risk for disseminated viral infections, such as is known to occur with varicella-zoster virus. Thus 
administration of high doses of corticosteroids can present a distinct hazard to a recipient, this being especially so with young children. A recent media report ${ }^{18}$ cites the use of prednisolone therapy in a child with ocular inflammation, who subsequently died from a fulminating varicella-zoster infection.

Although rarely undertaken nowadays, use of corticosteroids as monotherapy for treatment of active focal toxoplasmic retinochoroiditis shows that there is no unequivocal evidence of iatrogenic complications. ${ }^{19}$ There is, however, a theoretical risk of uncontrolled replication of Toxoplasma in the retinae of some patients, and this could lead to exacerbation of the disease process. By downregulating the inflammatory response without providing appropriate anti-protozoal cover, corticosteroid monotherapy could further increase the risk of recurrence of the infection, since the parasitic burden and the number of parasitised cells may be greater than if appropriate chemotherapeutic agents are provided. There are a number of anecdotal reports describing fulminant ocular toxoplasmosis when corticosteroids have been administered in the absence of anti-Toxoplasma chemotherapy. Periocular use of corticosteroids is also contraindicated, since this increases the risk of exacerbation of Toxoplasma infection. ${ }^{20}$

Consideration of how medical treatment of toxoplasmic retinochoroiditis should be rationalised must involve analysis of aspects relating to the cell biology of Toxoplasma ${ }^{21}$ and, more specifically, the mechanism(s) of interaction of candidate drugs which target organelles of the protozoan. ${ }^{22}$ Such an approach would include host immune effects, such as the possible role of nitric oxide in stage conversion from the endozoite (tachyzoite) to cystozoite (bradyzoite) form of the protozoan, ${ }^{23}$ as well as the phylogeny of Toxoplasma, which has suggested that the clonal lineage of pathogenic strains of the protozoan may have important implications for the development of drug treatments. ${ }^{24}$ Ultimately, however, the benefits of basic science studies and potential therapeutic value of such treatments in the ocular setting can be established only from internationally agreed classifications for both diagnosis and degree of severity of the disease process, taken in combination with findings from a largescale, longitudinal, randomised and masked multicentre drug-treatment trial.

JOHN HAY

GORDON N. DUTTON

Tennent Institute of Ophthalmology

Western Infirmary

Glasgow,

UK

\section{References}

1. Remington JS, McLeod R, Desmonts G. Toxoplasmosis. In: Remington JS, Klein JO, editors. Infectious diseases of the fetus and newborn infant. 4th ed. Philadelphia: WB Saunders, 1996:140-267.

2. Stanford M. Acute symptomatic Toxoplasma retinochoroiditis. Eye 1996;10:3-4.

3. McMenamin PG, Dutton GN, Hay J, Cameron S. The ultrastructural pathology of congenital murine toxoplasmic retinochoroiditis. I. The localization and morphology of Toxoplasma cysts in the retina. Exp Eye Res 1986;43:529-43.

4. Beaman MH, Wong S-Y, Remington JS. Cytokines, Toxoplasma and intracellular parasitism. Immunol Rev 1992;127:97-117.

5. Holland GN, O'Connor GR, Belford R Jr, Remington JS. Toxoplasmosis. In: Pepose JS, Holland GW, Wilhelmus KR, editors. Ocular infection and immunity. St Louis: Mosby, 1996:1183-223.

6. Rothova A. Ocular involvement in toxoplasmosis. Br J Ophthalmol 1993;77:371-7.

7. Engstrom RE, Holland GN, Nussenblatt RB, Jabs DA. Current practices in the management of ocular toxoplasmosis. Am J Ophthalmol 1991;115:601-10.

8. McCabe RE, Oster S. Current recommendations and future prospects in the treatment of toxoplasmosis. Drugs 1989;38:973-87.

9. Rothova A, Meenken C, Buitenhuis HJ, Brinkman CJ, Baarsma GS, Boen-Tan TN, de Jong PTVM, Khaassen-Brockema N, Schweitzer CMC, Timmerman Z, de Vries J, Zaal MJW, Kiljstra A. Therapy for ocular toxoplasmosis. Am J Ophthalmol 1993;115:517-23.

10. Mets MB, Holfels E, Boyer KM, Swisher CN, Roizen N, Stein L, Hopkins J, Withers S, Mack D, Luciano R, Patel D, Remington JS, Meier P, McLeod R. Eye manifestations of congenital toxoplasmosis. Am J Ophthalmol 1996;122:309-24.

11. Cochereau-Massin I, Le Hoang P, Lautier-Frau M, Zerdoun E, Zazoun L, Robinet M, Marcel P, Girard B, Katlama C, Leport C, Rozenbaum W, Coulaud JP, Gentilini M. Ocular toxoplasmosis in human immunodeficiency virus-infected patients. Am J Ophthalmol 1992;114:130-5.

12. Holland GN, Engstrom RE Jr, Glasgow BJ, Beuger BB, Daniels SA, Sidikaro Y, Harmon JA, Fischer DH, Boyer DS, Rao NA, Eagle RC Jr, Kreiger AE, Foos RY. Ocular toxoplasmosis in patients with the acquired immunodeficiency syndrome. Am J Ophthalmol 1988;106:653-67.

13. Sande MA, Whitley RJ, McCracken GH, Lentnek A, Scheld WM. Evaluation of new anti-infective drugs for the treatment of Toxoplasma encephalitis. Clin Infect Dis 1992;15(Suppl 1);S200-5.

14. Suzuki Y, Yang Q. Conley FK, Abrams JS, Remington JS. Antibody against interleukin-6 reduces inflammation and numbers of cysts in brains of mice with toxoplasmic encephalitis. Infect Immunol 1994; 62:2773-8.

15. Hunter CA, Subauste CS, Remington JS. The role of cytokines in toxoplasmosis. Biotherapy 1994;7:237-47.

16. Gazzinelli RT, Brezin A, Li Q, Nussenblatt RB, Chan C-C. Toxoplasma gondii: acquired ocular toxoplasmosis in the murine model, protective role of TNF- $\alpha$ and IFN- $\gamma$. Exp Parasitol 1994;78:217-29.

17. Cesario TC, Slater LM, Kaplan HS. Effect of antineoplastic agents on gamma-interferon production in human peripheral blood mononuclear cells. Cancer Res 1984;44:4962-6. 
18. Leake J. Thousands fight for steroid justice. The Sunday Times, 27 August 1995.

19. Acers TE. Toxoplasmic retinochoroiditis: a double blind therapeutic study. Arch Ophthalmol 1964; 71:58-62.

20. O'Connor GR, Frenkel JK. Dangers of steroid therapy in toxoplasmosis. Arch Ophthalmol 1976;94:213-6.

21. Dubremetz JF. Toxoplasma gondii: cell biology update. In: Boothroyd JC, Komuniecki R, editors. Molecular approaches to parasitology. New York: Wiley, 1995: 345-58.
22. Tomavo S, Boothroyd JC. Interconnection between organellar functions, development and drug resistance in the protozoan parasite, Toxoplasma gondii. Int $\mathbf{J}$ Parasitol 1995;25:1293-9.

23. James SL. Role of nitric oxide in parasitic infections. Microbiol Rev 1995;59:533-47.

24. Sibley LD, Howe DK. Toxoplasma gondii comprises three clonal lineages: correlation of parasite genotype with human disease. J Infect Dis 1995;172:1561-6. 